\title{
Tendencia de la incidencia de los tumores hepáticos en la infancia
}

Juan Manuel Mejía-A ranguré, $M$ en $C$, $^{(1)} O$ lbeth Beutelspacher-Vázquez, MD, ${ }^{(2)}$ Servando Juárez- 0 caña, $M$ en $C,{ }^{(1)}$ José Vázquez-Langle, MD, ${ }^{(3)}$ María del C armen Martínez-García, M en $C,{ }^{(1)} A$ rturo Fajardo-Gutiérrez, $M$ en $C$. $^{(1)}$

\section{Mejía-Aranguré JM, Beutelspacher-Vázquez 0 , Juárez-Ocaña S, Vázquez-Langle J, Martínez-García MC, Fajardo-Gutiérrez A. Tendencia de la incidencia de los tumores hepáticos en la infancia. Salud Publica Mex 2002;44:100-107.} El texto completo en inglés de este artículo está disponible en: http://www.insp.mx/salud/index.html

\section{Resumen}

Objetivo. Evaluar la tendencia de la incidencia de los diferentes tumores hepáticos en niños residentes en el D istrito Federal. Material y métodos Encuesta hospitalaria. Se realizó un análisis de dos bases de datos. La primera tiene registrados todos los casos de tumo res hepáticos que se encontraron entre el periodo de 1982 a 1991, de hospitales que atienden a niños con cáncer, residentes en la Ciudad de México. La segunda base de datos tiene registrados todos los casos de tumores hepáticos que se encontraron de 1996 a 1999 en el Hospital de Pediatría Centro Médico $\mathrm{N}$ acional (CMN) "Siglo XXI" y en el Hospital General del Centro Médico N acional "La Raza", del Instituto Mexicano del Seguro So cial (IMSS). Se calcularon las tasas de incidencia anual promedio (TIAP) por cada tipo de tumor hepático. Las tasas fueron estandarizadas por el método directo, usando como población de referencia a la mundial estándar menor de 15 años. La tendencia se evaluó con las tasas de incidencia anuales y se calculó la tasa de cambio promedio que emplea la distribución de Poisson. Resultados Durante el periodo de 1982 a 1991 la TIAP para hepato blastoma fue el triple en hombres con $0.6 \times 10^{6}$. El grupo más afectado fue el de 1 a 4 años. Para los hepatocarcinomas la TIAP fue de 0.14 para el sexo femenino, siendo el doble que la del sexo masculino. Para el periodo de 1996 a 1999 la

\author{
Mejía-Aranguré JM, Beutelspacher-Vázquez 0 , \\ Juárez-Ocaña S, Vázquez-Langle J, \\ Martínez-García MC, Fajardo-Gutiérrez A. \\ Incidence trends of hepatic \\ tumors in childhood. \\ Salud Publica Mex 2002;44:100-107. \\ The English version of this paper \\ is available at: http://www.insp.mx/salud/index.html
}

\begin{abstract}
A bstract
Objective. To evaluate the incidence trends of hepatic tumors among children living in Mexico C ity. Material and Methods. A cross-sectional ho spital survey was conducted to yield two databases. The first database contains the registry of all the cases of hepatic tumors occurring during the period 1982-1991, in public hospitals of Mexico City. The second database contains all hepatic tumor cases fo und between 1996 and 1999 in Hospital de Pediatría del Centro Médico N acional "Siglo XXI" and in Hospital General del C entro Médico La Raza, both hospitals pertaining to Instituto Mexicano del Seguro Social (Mexican Institute of Social Security). The average annual incidence rates (AAIR) were calculated for each type of hepatic tumor. The rates were standardized with the direct method, using as standard the world population under 15 years of age. The trends were evaluated with the annual incidence rates and the average rate of change assuming a Poisson distribution. Results. The AAIR for hepatoblastoma during the period 1982-1991 was three times higher for men than for women, with a value of $0.6 \times 10^{6}$. The group of 1-4 years of age was the most affected. For hepatocarcinomas the AAIR was twofold for women (0.14) as compared to men. Between 19961999 the AAIR for hepatoblastoma was 5.11 in women and 1.85 in men.The age group with the highest rate was women
\end{abstract}

Este estudio fue parcialmente apoyado por los financiamientos del Instituto Mexicano del Seguro Social (FP-00038/218/415/459).

(1) Unidad de Investigación Médica en Epidemiología Clínica, Hospital de Pediatría, Centro Médico N acional "Siglo XXI", Instituto Mexicano del Seguro Social (IMSS), México, D.F., México.

(2) Servicio de Urgencias, Hospital General de Zona N 0. 30, IMSS, México, D.F., México.

(3) Servicio de Cirugía de Alta Especialidad, Hospital de Pediatría, Centro Médico N acional "Siglo XXI", IMSS, México, D.F., México.

Solicitud de sobretiros: Dr. Juan Manuel Mejía-Aranguré. Sección de Epidemiología Molecular, Unidad de Investigación Médica en Epidemiología Clínica, Hospital de Pediatría 3er Piso. Cuauhtémoc 330, Col. D octores 06725. México, D F, México.

Correo electrónico: arangurejm@ hotmail.com 
TIAP para hepatoblastomas fue de 5.11 en mujeres y de 1.85 en hombres. El grupo de edad con la tasa más alta fue el de mujeres menores de un año. Para hepatocarcinomas laTIAP fue de 0.64 para hombres y de 1.23 en mujeres. El grupo de edad más afectado fue el de hombres de 10 a 14 años. No se observó tendencia significativa al incremento o decremento en la incidencia de hepatoblastomas. Para hepatocarcinomas hubo una tasa de cambio de $10 \%$, pero tampoco fue significativa. Conclusiones. No existe en la Ciudad de México una tendencia en la incidencia de los tumores hepáticos en niños menores de 15 años, de 1982 a 1991, y de 1996 a 1999. El texto completo en inglés de este artículo está disponible en:http://www.insp.mx/salud/ index.html

Palabras clave: hepato blastoma/tendencias; carcinoma hepatocelular/tendencias; incidencia; México under one year of age.The AAIR for hepatocarcinoma was 0.64 for males and 1.23 for females. The most affected age group was males aged 10 to 14 years. No significant upward or downward trend was found in the incidence of hepatoblasto mas. A non-significant change rate of $10 \%$ was found for hepatocarcinoma. Conclusions No significant trends were observed in the incidence of hepatic tumors in children of Mexico City aged under 15 years, during the periods 1982-1991 and 1996-1999. The English version of this paper is available at:http://ww w.insp.mx/salud/index.html

Key words: hepatoblastoma/trends; carcinoma, hepatocellular/trends; incidence; Mexico os tumores hepáticos primarios malignos representan sólo de 1.5 a 5\% de todas las neoplasias en la edad pediátrica, y se presentan con mayor frecuencia que los tumores benignos. Existen dos formas principales de tumores hepáticos malignos: el hepatoblastoma y el carcinoma hepatocelular. El 90\% lo constituyen los hepatoblastomas. ${ }^{1,2}$ Aproximadamente, 80\% de los casos de hepatoblastoma se presenta en menores de tres años de edad. ${ }^{3}$ El hepatocarcinoma es un tumor epitelial maligno, mucho menos frecuente que el hepatoblastoma y se presenta en edades más tardías, como promedio a los nueve años. ${ }^{4}$

Los tumores de hígado son más frecuentes en Asia y Africa, y son el tercer tumor más común en niños japoneses. ${ }^{5} \mathrm{La}$ incidencia de hepatoblastoma alrededor del mundo permanece constante entre 0.5 y 1.5 casos x $10^{6}$ de niños. ${ }^{6}$ En la mayoría de los países el carcinoma hepatocelular es menos común que el hepatoblastoma, pero hay una variación geográfica considerable con cifras desde 0.2 x $10^{6}$ en Inglaterra y Gales, hasta 2.1 x $10^{6}$ de niños en Hong Kong. En algunas poblaciones el hepatocarcinoma se presenta más frecuentemente que el hepatoblastoma, como en el caso de Hong Kong y Taiwán. ${ }^{7}$

En los Estados Unidos de América se reporta para el hepatoblastoma una incidencia anual de aproximadamente $1 \times 10^{6}$ en niños menores de 15 años de edad. ${ }^{8}$ Los datos sobre la frecuencia de los tumores hepáticos en México son aislados y parciales. Sólo se han comunicado algunos datos sobre mortalidad y frecuencia de las diferentes neoplasias en hospitales que atienden a niños con tumores malignos. ${ }^{9,10}$ En un estudio multicéntrico realizado en nuestro país se encontraron 58 casos de hepatoblastoma y 10 de hepatocarcinoma durante el periodo comprendido entre 1980 y 1991, y correspondieron a todos los casos informados de niños menores de 15 años, atendidos en hospitales de la Ciudad de México. ${ }^{11}$ En ningún informe nacional se ha dado la tasa de incidencia de los diferentes tipos de tumores hepáticos, ni se ha señalado si existe una tendencia en la frecuencia en los últimos años.

\section{Material y métodos}

Se hizo un análisis de dos bases de datos. La primera tiene registrados todos los casos de tumores hepáticos que se encontraron entre el periodo de 1982 a 1991 en los siguientes hospitales: 1) Hospital Infantil de México "Federico Gómez", de la Secretaría de Salud (SSA), 2) Hospital de Pediatría del Centro Médico Nacional "Siglo XXI" del Instituto Mexicano del Seguro Social (IMSS), 3) Hospital 20 de Noviembre del Instituto de Seguridad y Servicios Sociales de los Trabajadores del Estado (ISSSTE), 4) Hospital General de México de la SSA; 5) Hospital General del Centro Médico "La Raza” (IMSS), y 6) Instituto Nacional de Pediatría. En estos hospitales se sabe que se atiende $95 \%$ de todos los casos con cáncer de los niños residentes en la Ciudad de México. ${ }^{11}$

La estandarización del personal para la captura de la información ha sido previamente reportada. ${ }^{12}$

La segunda base de datos tiene registrados todos los casos de tumores hepáticos que se encontraron entre el periodo de 1996 a 1999 en el Hospital de Pediatría del CMN "Siglo XXI", y en el Hospital General del Centro Médico Nacional "La Raza", hospitales del tercer nivel de atención que ven prácticamente a todos los niños con tumores hepáticos malignos, derechohabientes del IMSS residentes en el Distrito Federal. 
Las variables estudiadas para este estudio fueron tipo de tumor hepático, edad, sexo, lugar de residencia, reporte histológico y peso al nacimiento. Sólo se seleccionaron los niños residentes en el Distrito Federal.

El periodo del análisis para la primera base de datos fue de 1982 a 1991, y para la segunda de 1996 a 1999.

La población de referencia para calcular las tasas de incidencia de 1982 a 1991 fue la de niños menores de 15 años residentes en la Ciudad de México durante el periodo de estudio. Estos datos se obtuvieron del Instituto Nacional de Estadística, Geografía e Informática (INEGI). La población de referencia para calcular las tasas de incidencia de 1996 a 1999 se obtuvo de la Coordinación de Planeación e Información Médica del IMSS. Se usó como denominador la población menor de 15 años adscrita a médico familiar durante los años correspondientes. La población de hombres fue de 400 795, 423 335, 389 520, 410903 para los años 1996, 1997, 1998 y 1999 respectivamente. Para mujeres la población fue de 385 959, 408 864, 375 748 y 395140 para los años 1996, 1997, 1998 y 1999, respectivamente.

Se calcularon las tasas de incidencia anuales promedio (TIAP), por cada tipo de tumor hepático. Esta tasa se calculó para cada año de estudio y se reportó por $10^{6}$. Las tasas para ambos periodos de estudio fueron estandarizadas por el método directo, usando como población de referencia a la población mundial estándar menor de 15 años. ${ }^{12}$

Para evaluar la tendencia para los periodos de estudio se analizaron las tasas de incidencia anuales y para identificar la magnitud del cambio de las tasas se calculó la tasa de cambio promedio para dicho periodo, usando un modelo de máxima verosimilitud, basado en una distribución de Poisson. ${ }^{13}$ Además, se calcularon los intervalos de confianza a $95 \%$ de las tasas de cambio. Esta técnica se recomienda para medir algún incremento o decremento en la frecuencia de la enfermedad a lo largo del tiempo. ${ }^{13}$

\section{Resultados}

Durante el periodo de 1982 a 1991 se encontraron 16 casos de tumores hepáticos; 13 correspondieron a hepatoblastomas y tres a hepatocarcinomas, correspondiendo una (TIAP) de $0.5 \times 10^{6}$ con una tasa estandarizada de $0.60 \times 10^{6}$. Hubo cuatro niñas con hepatoblastoma y dos con hepatocarcinoma. Tomando en cuenta sólo la población del IMSS, en este periodo se registraron cinco casos de tumores hepáticos, los cuales fueron hepatoblastomas. Las tasas fueron de 0.81 , de $0.85,0.82,0.91$ y $0.87 \times 10^{6}$, para los años de 1986 y de 1988 a 1991, respectivamente. Como en los demás años no hubo casos en la población del IMSS, no se reportó una tasa de incidencia. Con estos resultados se obtuvo una TIAP de tumores hepáticos para la población del IMSS residente en la Ciudad de México, durante este periodo de estudio de $0.40 \times 10^{6}$. Apenas inferior a la TIAP de la Ciudad de México de tumores hepáticos en general, e idéntica a la de hepatoblastomas.

La TIAP para hepatoblastoma fue mayor en el sexo masculino de $0.6 \times 10^{6}$. En uno u otro sexo se encontraron las TIAP más altas en el grupo de menores de un año (cuadro I), seguidas por el grupo de 1 a 4 años. Es notorio que para el grupo de 5 a 9 años las TIAP son iguales para uno $\mathrm{u}$ otro sexo.

No se observó una tendencia significativa hacia el incremento o decremento en la incidencia de hepatoblastomas. En el sexo femenino la tasa de cambio anual fue negativa, lo cual hablaría de que se observó una tendencia al decremento de la incidencia, pero no fue significativa, como se aprecia por los intervalos de confianza. En el sexo masculino la tasa de cambio mostró una ligera tendencia al incremento de $1 \%$, pero ésta tampoco fue significativa.

En relación con los hepatocarcinomas se observó una TIAP de $0.14 \times 10^{6}$ en el sexo femenino, que fue el doble de la del sexo masculino (cuadro II). En el sexo femenino la tasa más alta se encontró en el grupo de 1 a 4 años, y para el masculino en el de menores de un año. La tasa de cambio para los hepatocarcinomas en general mostró una variación de 10\%, lo cual señalaría cierta tendencia al incremento; sin embargo, ésta no fue significativa. En el grupo de pacientes masculinos fue donde se observó la mayor tasa de cambio, $6 \%$, pero ésta no puede ser del todo valorable, puesto que sólo se presentó un caso durante el periodo de 1982 a 1991. En este periodo no se reportaron casos de hepatocarcinomas en los hospitales del IMSS.

Durante el periodo de 1996 a 1999 se encontró una TIAP para los tumores hepáticos en general de $4.39 \mathrm{x}$ $10^{6}$, y una tasa estandarizada de $5.12 \times 10^{6}$. Esta tasa fue notoriamente mayor a la reportada durante el periodo de 1982 a 1991. Hubo 14 casos durante este periodo, nueve de ellos del sexo femenino; 11 casos tuvieron el diagnóstico de hepatoblastoma (cuadro III), ocho de ellos en mujeres.

La TIAP fue mayor en el sexo femenino para los hepatoblastomas (cuadro III). En el sexo femenino la TIAP más alta se encontró en el grupo de menores de un año; no se encontró ningún caso de esta edad en el sexo masculino. En el grupo de 1 a 4 años se encontraron todos los casos de hepatoblastoma en el sexo masculino. La tasa de cambio anual fue menor de $1 \%$, tanto 


\section{Cuadro I}

INCIDENCIA DE HEPATOBLASTOMA EN NIÑOS MENORES DE 15 AÑOS RESIDENTES en la Ciudad de México, 1982 a 1991

\begin{tabular}{|c|c|c|c|c|c|c|c|c|c|c|c|c|c|c|c|c|}
\hline Año & 82 & 83 & 84 & 85 & 86 & 87 & 88 & 89 & 90 & 91 & Casos & TIAP & TST & TC\% & Lim. Inf. & Lim. Sup. \\
\hline Grupo total & 0 & 1.3 & 0.3 & 0.3 & 0.7 & 0.3 & 0.3 & 0.3 & 0.4 & 0.4 & 13 & 0.4 & 0.53 & -1.62 & -19.59 & 20.37 \\
\hline Mujeres total & 0 & 1.3 & 0 & 0.6 & 0 & 0 & 0.7 & 0 & 0 & 0 & 4 & 0.2 & 0.325 & -2.45 & -14.4 & 11.16 \\
\hline$<1$ año & 0 & 10.5 & 0 & 0 & 0 & 0 & 0 & 0 & 0 & 0 & 1 & 1.13 & & & & \\
\hline 1 a 4 años & 0 & 2.53 & 0 & 2.66 & 0 & 0 & 0 & 0 & 0 & 0 & 2 & 0.55 & & & & \\
\hline 5 a 9 años & 0 & 0 & 0 & 0 & 0 & 0 & 2.26 & 0 & 0 & 0 & 1 & 0.21 & & & & \\
\hline 10 a 14 años & 0 & 0 & 0 & 0 & 0 & 0 & 0 & 0 & 0 & 0 & 0 & 0 & & & & \\
\hline Tasa estandarizada & 0 & 1.57 & 0 & 0.8 & 0 & 0 & 0.7 & 0 & 0 & 0 & & & & & & \\
\hline Hombres total & 0 & 1.3 & 0.6 & 0 & 1.4 & 0.7 & 0 & 0.7 & 0.7 & 0.8 & 9 & 0.6 & 0.732 & 1.01 & -10.78 & 14.37 \\
\hline$<1$ año & 0 & 0 & 10.4 & 0 & 0 & 0 & 0 & 0 & 0 & 12.3 & 2 & 2.18 & & & & \\
\hline 1 a 4 años & 0 & 2.47 & 0 & 0 & 5.33 & 2.73 & 0 & 2.89 & 2.97 & 0 & 6 & 1.6 & & & & \\
\hline 5 a 9 años & 0 & 1.87 & 0 & 0 & 0 & 0 & 0 & 0 & 0 & 0 & 1 & 0.21 & & & & \\
\hline 10 a 14 años & 0 & 0 & 0 & 0 & 0 & 0 & 0 & 0 & 0 & 0 & 0 & 0 & & & & \\
\hline Tasa estandarizada & 0 & 1.32 & 0.8 & 0 & 1.64 & 0.83 & 0 & 0.86 & 0.89 & 0.95 & & & & & & \\
\hline
\end{tabular}

TIAP: tasa incidencia anual promedio

TST : tasa estandarizada

TC\%: tasa de cambio

Lim. Inf.: límite inferior de intervalo de confianza al 95\% de laTC

Lim. Sup.: límite superior de intervalo de confianza al $95 \%$ de laTC

\section{Cuadro II}

\section{INCIDENCIA DE HEPATOCARCINOMA EN NIÑOS MENORES DE 15 AÑOS RESIDENTES} en la Ciudad de México, 1982 a 1991

\begin{tabular}{|c|c|c|c|c|c|c|c|c|c|c|c|c|c|c|c|c|}
\hline Año & 82 & 83 & 84 & 85 & 86 & 87 & 88 & 89 & 90 & 91 & Casos & TIAP & TST & TC\% & Lim. Inf. & Lim. Sup \\
\hline Grupo total & 0 & 0 & 0 & 0 & 0.3 & 0 & 0.3 & 0 & 0 & 0.4 & 3 & 0.11 & 0.074 & 10.56 & -27.86 & 69.45 \\
\hline Mujeres total & 0 & 0 & 0 & 0 & 0.7 & 0 & 0.7 & 0 & 0 & 0 & 2 & 0.14 & 0.147 & 1.3 & -18.96 & 26.62 \\
\hline$<1$ año & 0 & 0 & 0 & 0 & 0 & 0 & 0 & 0 & 0 & 0 & 0 & 0 & & & & \\
\hline 1 a 4 años & 0 & 0 & 0 & 0 & 0 & 0 & 2.8 & 0 & 0 & 0 & 1 & 0.27 & & & & \\
\hline 5 a 9 años & 0 & 0 & 0 & 0 & 0 & 0 & 0 & 0 & 0 & 0 & 0 & 0 & & & & \\
\hline 10 a 14 años & 0 & 0 & 0 & 0 & 2.1 & 0 & 0 & 0 & 0 & 0 & 1 & 0.22 & & & & \\
\hline Tasa estandarizada & 0 & 0 & 0 & 0 & 0.6 & 0 & 0.86 & 0 & 0 & 0 & & & & & & \\
\hline Hombres total & 0 & 0 & 0 & 0 & 0 & 0 & 0 & 0 & 0 & 0.8 & 1 & 0.07 & 0.084 & 6.13 & -15.56 & 33.39 \\
\hline$<1$ año & 0 & 0 & 0 & 0 & 0 & 0 & 0 & 0 & 0 & 12.3 & 1 & 1.09 & & & & \\
\hline 1 a 4 años & 0 & 0 & 0 & 0 & 0 & 0 & 0 & 0 & 0 & 0 & 0 & 0 & & & & \\
\hline 5 a 9 años & 0 & 0 & 0 & 0 & 0 & 0 & 0 & 0 & 0 & 0 & 0 & 0 & & & & \\
\hline 10 a 14 años & 0 & 0 & 0 & 0 & 0 & 0 & 0 & 0 & 0 & 0 & 0 & 0 & & & & \\
\hline Tasa estandarizada & 0 & 0 & 0 & 0 & 0 & 0 & 0 & 0 & 0 & 0.95 & & & & & & \\
\hline $\begin{array}{l}\text { TIAP: tasa incidencia anu } \\
\text { TST: tasa estandarizada } \\
\text { TC\%: tasa de cambio } \\
\text { Lim. Inf.: límite inferior d } \\
\text { Lim. Sup.: límite superior }\end{array}$ & & & & & & & & & & & & & & & & \\
\hline
\end{tabular}




\section{Cuadro III \\ Incidencia de hepatoblastoma en niños menores de 15 años derechohabientes del Instituto Mexicano del Seguro Social residentes en la Ciudad de México, 1996 a 1999}

\begin{tabular}{|c|c|c|c|c|c|c|c|c|c|c|}
\hline Año & 96 & 97 & 98 & 99 & Casos & TIAP & TST & TC\% & Lim. Inf. & Lim. Sup. \\
\hline Grupo total & 7.63 & 2.4 & 2.61 & 1.24 & 11 & 3.45 & 4.26 & -0.4 & -4.36 & 3.72 \\
\hline Mujeres total & 12.95 & 2.45 & 2.66 & 2.5 & 8 & 5.11 & 6.58 & -0.16 & -1.98 & 1.7 \\
\hline$<1$ año & 135.4 & 0 & 49.83 & 48.81 & 5 & 58.82 & & & & \\
\hline 1 a 4 años & 9.53 & 8.82 & 0 & 0 & 2 & 4.65 & & & & \\
\hline 5 a 9 años & 7.57 & 0 & 0 & 0 & 1 & 1.84 & & & & \\
\hline 10 a 14 años & 0 & 0 & 0 & 0 & 0 & 0 & & & & \\
\hline Tasa estandarizada & 15.9 & 2.73 & 3.85 & 3.77 & & & & & & \\
\hline Hombres total & 2.5 & 2.36 & 2.57 & 0 & 3 & 1.85 & 2.06 & -0.72 & -8.57 & 7.79 \\
\hline$<1$ año & 0 & 0 & 0 & 0 & 0 & 0 & & & & \\
\hline 1 a 4 años & 9.12 & 8.46 & 9.25 & 0 & 3 & 6.68 & & & & \\
\hline 5 a 9 años & 0 & 0 & 0 & 0 & 0 & 0 & & & & \\
\hline 10 a 14 años & 0 & 0 & 0 & 0 & 0 & 0 & & & & \\
\hline Tasa estandarizada & 2.82 & 2.61 & 2.86 & 0 & & & & & & \\
\hline
\end{tabular}

TIAP: tasa incidencia anual promedio

TST: tasa estandarizada

TC \%: tasa de cambio

Lim. Inf:: límite inferior de intervalo de confianza al 95\% de laTC

Lim. Sup.: límite superior de intervalo de confianza al 95\% de laTC

en hombres como en mujeres, lo cual indica que no hay aparentes cambios en la tendencia de la incidencia del hepatoblastoma en esos cuatro años de estudio.

Se encontró una incidencia más alta de hepatocarcinomas en el sexo masculino (cuadro IV). Sólo se encontró un caso de hepatocarcinoma en el sexo femenino en el grupo entre 5 a 9 años. Hubo dos casos en el sexo masculino en el grupo entre 10 a 14 años. La tasa de cambio anual para uno u otro sexo fue menor de $1 \%$ y no fue significativa, lo cual nos indica que con esta enfermedad no ha habido cambios en la incidencia.

\section{Discusión}

En el presente estudio se analizaron dos diferentes bases de datos. Las tasas estandarizadas para tumores hepáticos fueron muy distintas, dependiendo de la base analizada. Mientras que con la base de datos de todos los hospitales de la Ciudad de México se encontró una tasa de $0.6 \times 10^{6}$, con la de los hospitales del IMSS se encontró una tasa estandarizada de 5.12. Estas diferencias pueden deberse a que la base de datos de todos los hospitales de la Ciudad de México consistió en una revisión retrospectiva de expedientes, lo que pudo traer como consecuencia una pérdida de casos.
Mientras que la base de datos de los hospitales del IMSS, que comprendió de 1996 a 1999, fue prospectiva, pues ha venido captando todos los nuevos casos que se informan de estos hospitales; por lo que es poco probable que la base de datos haya tenido pérdida de casos.

Aunque la evaluación de la primera base de datos (1982 a 1991) proporciona una TIAP subestimada, permite vislumbrar la tendencia de la incidencia de este padecimiento durante los años evaluados. Vale la pena también mencionar que cuando se evaluó la tasa de incidencia de tumores hepáticos en general, sólo en la población del IMSS durante el periodo de 1982 a 1991, la tasa fue muy similar a la reportada para toda la Ciudad de México en el mismo periodo. Lo que hace pensar que dicha subestimación se dio en forma general en todas las instituciones incluidas en el estudio.

Por otro lado, puesto que las TIAP para la ciudad de México y el IMSS son similares durante este periodo, se consideró pertinente discutir sólo los datos de la Ciudad de México por su mayor representatividad.

A pesar de la subestimación de la tasa de incidencia para este periodo, se encontró una tasa dentro de los rangos conocidos para la incidencia de esta enfermedad en el mundo, que va de 0.2 a $3.8 \times 10^{6}$; para 


\section{Cuadro IV \\ INCIDENCIA DE HEPATOCARDINOMA EN NIÑOS MENORES DE 15 AÑOS DERECHOHABIENTES del Instituto Mexicano del Seguro Social residentes en la Ciudad de México, 1996 a 1999}

\begin{tabular}{lrrrrrrrrrr} 
Año & 96 & 97 & 98 & 99 & Casos & TIAP & TST & TC\% & Lim. Inf. & Lim. Sup. \\
Grupo total & 1.27 & 0 & 1.31 & 1.24 & 3 & 0.94 & 0.85 & 0.52 & -16.05 & 20.35 \\
\hline Mujeres total & 0 & 0 & 0 & 2.5 & 1 & 0.64 & 0.59 & 0.89 & -8.6 & 11.36 \\
\hline$<1$ año & 0 & 0 & 0 & 0 & 0 & 0 & & & \\
\hline 1 a 4 años & 0 & 0 & 0 & 0 & 0 & 0 & & & \\
\hline 5 a 9 años & 0 & 0 & 0 & 7.19 & 1 & 1.84 & & & -59.94 \\
\hline 10 a 14 años & 0 & 0 & 0 & 0 & 0 & 0 & & & 129.14 \\
\hline Tasa estandarizada & 0 & 0 & 0 & 2.31 & & & & -0.43 & -1.11 \\
Hombres total & 2.5 & 0 & 2.57 & 0 & 2 & 1.23 & & \\
\hline$<1$ año & 0 & 0 & 0 & 0 & 0 & 0 & & & \\
\hline 1 a 4 años & 0 & 0 & 0 & 0 & 0 & 0 & & & \\
\hline 5 a 9 años & 0 & 0 & 0 & 0 & 0 & 0 & & & \\
\hline 10 a 14 años & 7.65 & 0 & 8.05 & 0 & 2 & 3.83 & & &
\end{tabular}

TIAP: tasa incidencia anual promedio

TST: tasa estandarizada

TC \%: tasa de cambio

Lim. Inf.: límite inferior de intervalo de confianza al 95\% de laTC

Lim. Sup.: Iímite superior de intervalo de confianza al 95\% de laTC

los tumores hepáticos en general y de 0.2 a $2.7 \times 10^{6}$, para los hepatoblastomas, y de 0 a 1.8 para los hepatocarcinomas. ${ }^{14}$ De igual forma es coincidente en la mayoría de los países el no haber encontrado tendencia al incremento o decremento de este padecimiento. Es importante comentar que en la mayor parte del mundo no se ha informado de incremento o decremento en la incidencia de estas neoplasias, lo cual resalta la utilidad de este reporte. ${ }^{15}$

Algo que llama muchísimo la atención es el haber encontrado un caso de hepatocarcinoma en un niño menor de un año, ya que esta enfermedad se presenta sobre todo en los mayores de nueve años. ${ }^{4}$ No hay una explicación para este hallazgo pero se considera importante recalcarlo. En Costa Rica también fue hallado un caso de hepatocarcinoma en un niño menor de un año en el periodo de 1984 a 1992. ${ }^{14}$ En Cuba también fue reportado (1986 a 1990) un caso de hepatocarcinoma, sólo que en esta ocasión correspondió al sexo femenino. ${ }^{14}$ En otros tres registros de los Estados Unidos de América han sido reportados casos de hepatocarcinomas en menores de un año y todos ellos en el sexo masculino; los registros fueron Greater Delaware Valley Tumor Registry (1980-1999); New York State Cancer Registry (1983-1991); Surveillance, Epidemio- logy and END Results Program (SEER) Program (19831992). ${ }^{14}$

En relación con la segunda base de datos (1996 a 1999) llama bastante la atención la tasa tan elevada de tumores hepáticos, de $5.12 \times 10^{6}$ contra 3.8 , que es la tasa más alta que se ha reportado. ${ }^{14}$ Aquí lo que puede estar influyendo es el periodo tan corto que se está evaluando.

La tasa estandarizada de hepatoblastomas también fue más alta a la reportada mundialmente, sobre todo en el sexo femenino. Si bien no hay explicaciones para este fenómeno, algo que llama la atención es que los individuos de origen hispano, del registro Los Angeles County Surveillance Program (1984-1992), presentan la tasa de incidencia más alta de hepatoblastomas, alcanzando una tasa de $6.7 \times 10^{6}$ en el grupo de 0 a 4 años. ${ }^{14}$

Durante el periodo de 1996 a 1999 tampoco se observaron cambios en la incidencia de esta enfermedad, como ha sido mencionado por otros autores. ${ }^{15}$ En algunas series en donde se ha observado un cambio en la incidencia se ha señalado que esto puede deberse a errores en la clasificación histológica. ${ }^{16}$

Algo muy notorio es que mientras que en el periodo de 1982 a 1991 la razón hombre-mujer para el 
hepatoblastoma fue de 1.38, para el periodo de 1996 a 1999 fue de 0.31 . En la literatura mundial ${ }^{15,16}$ en casi todos los países se ha encontrado que el hepatoblastoma es más frecuente en los hombres que en las mujeres. En América sólo en el Ecuador se ha reportado una razón hombre-mujer de 0.3 , similar a la reportada en este estudio. ${ }^{14}$

Situación contraria ocurrió con los casos de hepatocarcinomas, en donde durante el periodo de 1982 a 1991 hubo más casos en mujeres (razón hombre-mujer 0.5) y durante el periodo de 1996 a 1999 hubo más casos en hombres que en mujeres (razón hombre-mujer de 1.88). En los diferentes reportes mundiales no se ha encontrado diferencia por sexo en esta enfermedad. ${ }^{16}$ En América sólo en Cuba se ha reportado una mayor frecuencia de hepatocarcinomas en las mujeres, con una razón hombre-mujer de 0.5. ${ }^{14}$

En algunos estudios se ha señalado la probabilidad de que el hepatoblastoma tenga su origen en la organogénesis y que esto pueda conducir a que los niños prematuros tengan un mayor riesgo de padecer esta enfermedad. ${ }^{17}$ En este estudio no se evaluó la edad gestacional o el peso al nacimiento de los casos y quizá sea importante en futuras investigaciones identificar si los niños de bajo peso tienen un mayor riesgo de padecer hepatoblastomas, y si en ellos sí ha habido un incremento en la incidencia del padecimiento. 8,17,18

La etiología del hepatoblastoma permanece aún desconocida. La mayoría de los niños con hepatoblastoma se encuentra dentro de los primeros tres años de edad, lo que sugiere la presencia de un componente genético. Se han reportado anormalidades cromosómicas en muchos pacientes con hepatoblastoma. La anomalía más frecuente parece ser la trisomía del cromosoma 20, seguida de trisomía del cromosoma 2. Estudios han demostrado anormalidades en el brazo corto del cromosoma 11, en la región del gene del factor 2 de crecimiento parecido a la insulina. ${ }^{3}$ Se han observado otras anomalías congénitas en pacientes con hepatoblastomas tales como hemihipertrofia, síndrome de Beckwith-Wiedemann, poliposis adenomatosa familiar, hernias diafragmática y umbilical. ${ }^{19}$ También se han reportado posibles asociaciones entre el hepatoblastoma y el uso de anticonceptivos orales, abuso de alcohol durante el embarazo, así como el tratamiento hormonal contra la esterilidad. ${ }^{20-22}$ También un estudio de casos y controles mostró que la exposición ocupacional materna a metales, productos derivados del petróleo, a pinturas y pigmentos, así como la exposición paterna a metales fueron factores de riesgo. ${ }^{23}$

En contraste, el hepatocarcinoma se presenta en niños mayores con una incidencia entre los 10 y 14 años de edad. ${ }^{24}$ Más de $30 \%$ de los pacientes con hepato- carcinoma presenta cirrosis hepática que puede ser secundaria a alteraciones metabólicas, como tirosinemia, galactosemia, deficiencia de a- 1 antitripsina, enfermedad por atesoramiento de glucógeno, cistinosis, enfermedad de Wilson, cistinuria, y enfermedad de Soto. ${ }^{5}$ Chen y colaboradores, en 1988 , demostraron $100 \%$ de positividad para HBsAg (antígenos de superficie para la hepatitis B) en 25 niños con hepatocarcinoma celular en Taiwán. ${ }^{7}$ El sureste de Asia es una área de alta prevalencia de infección con virus de la hepatitis B, pero el HBsAg en asociación con carcinoma hepatocelular en niños también ha sido reportada en Europa. ${ }^{25}$

Es importante identificar los factores asociados con el desarrollo de estos padecimientos, pues lo que por el momento puede decirse es que los factores que puedan estar ocasionando estas enfermedades no han repercutido para incrementar la frecuencia de este padecimiento en los periodos estudiados.

\section{Agradecimientos}

Agradecemos al técnico José Efrén Hernández en la preparación del presente manuscrito, y a la Enf. Guadalupe González por la recopilación de la información, así como sus valiosos comentarios sobre el presente manuscrito.

\section{Referencias}

1.- Exelby PR, Filler RM, G nosfeld JL. Liver tumor in children with particular reference to hepatoblastoma and hepatocellular carcinoma:A merican A cademy of Pediatric Surgical Section Survey-1974. Pediatr Surg 1975;10:329-337.

2.- Perilongo G, Shafford EA. Liver tumors. Eur J C ancer 1999;35:963-968.

3.- Raney B. Hepatoblastoma in Children: A Review. J Pediatr Hematol O ncol 1997;19:418-422.

4.- N ewman KD. Hepatic tumors in children. Sem Pediatr Surg 1997;6: 38-41.

5.- D ouglass EC . Liver tumors. Rudolph AM, H offman JIE, Rudolph CD, ed. Rudolph's Pediatrics. $19^{\text {th }}$ Edition. East N orwalk Connecticut:Appleton \& Lange, 1991:1213-1215.

6.- Parkin DM, Stiller CA, D raper GJ. Bieber CA.The international incidence of childhood cancer. Int I C ancer 1988;42:511-520.

7.- Chen W J, Lee JC, HungW T. Primary malignant tumor of liver in infants and children in Taiwan. J Pediatr Surg 1988;23:457-461.

8.- Ross JA, Gurney JG. Hepatoblastoma incidence in the United States from 1973 to 1992. Med Pediatr O ncol 1988:30:141-142.

9.- Rivera-Luna R, Martínez-Guerrero G. The problem of childhood cancer in a developing country. Int Pediatr Assoc 1986;7:411-419.

10.- Salas-Martínez M. Tumores hepáticos. N eoplasisas malignas en los niños. México: Editorial Panamericana, 1988:3-16.

11.- Fajardo-Gutiérrez A, Mendoza-Sánchez H,Valdez-Martínez E, MejíaA ranguré JM,Yamamoto-Kimura LT, Mejía D o mínguez A M et al. Frecuencia de neoplasias malignas en niños atendidos en hospitales del Distrito Federal. Estudio multicéntrico. Bol Med Hosp Infant Mex 1996;53:57-66. 
12.- Fajardo-G utiérrez A, Mejía-A ranguré JM, Gómez-D elgado A, Mendoza-Sánchez H, Garduño-Espinosa J, Martínez-García MC. Epidemiología de las neoplasias malignas en niños residentes del Distrito Federal (19821991). Bol Med Hosp Infant Mex 1995;52:507-516.

13.- Estève J, Benhamou E, Raymond L. Space Variations and group correlations. Statistical methods in cancer research. Descriptive Epidemiology of Childhood C ancer. Lyon, France: International A gency for Research on Cancer, 1994;IARC Publication N 0. 128:107-201.

14.- Schymura MJ, Kahn AR, W olfgang PE. Hepatic tumors. Parkin DM, Kramárová E, D raper GJ, Masuyer E, Michaelis JM,N egliaJ et al.International Incidence of Childhood Cancer. Vol II. IARC Scientific Publication. Lyon, France: International A gency for Research on Cancer 1998;N umber 144:125-130

15- Sharp L, Cotton S, Little J. Descriptive epidemiology. En: Little J, ed. Epidemiology of childhood cancer. Lyon, France: International A gency for Research on Cancer, 1999;IARC Scientific Publications N 0. 149:10-16.

16.- Bulterys M, Goodman MT, Smith M, Buckley J. Hepatic Tumors. En: Ries LAG, Smith MA, Gurney JG, Linet M, Tamra T, Young JL et al eds. Cancer Incidence and Survival among $C$ hildren and Adolescents: United States SEER Program 1975-1995, Bethesda, (MD): N ational Cancer Institute, SEER Program, 1999;N IH Pub. N 0. 99-44-49:91-98.

17.- Ikeda H, Matsuyama S,Tanimura M. Association between hepatoblastoma and very low birth weight:A trend or a chance? Pediatr 1997;130: 557-560.
18.- Young JL, Miller RW. Incidence of malignant tumors in U.S. children. J Pediatr 1975;86:254-258.

19.- Iwama T, Mishima V. Mortality in young first degree relatives of patients with familiar adenomatous polyposis. Cancer 1994;73:2065-2068.

20.- 0 tten J, Smets R, D e Jager R, Gerard A, Maurus R. Hepatoblastoma in an infant after contraceptive intake during pregnancy (letter). N Engl J Med 1977;297:222.

21.- Khan A, Bader JL, H oy G R, Sinks LF. H epatoblasto ma in child with fetal alcohol syndrome. Lancet 1979;1:403-404.

22.- Melamed I, Bujanover Y, Hammer J, Spirer Z. Hepatoblastoma in an infant born to a mother after hormonal treatment for sterility (letter). N Engl J Med 1982;307:820.

23.- Buckley JD, Sather H, Ruccione K, Rogers PCJ, H aas JE, Henderson BE et al. A case-control study of risk factors for hepatoblastoma. Cancer 1989;64:1169-1176

24.- Ishak KG, G lunz PR. H epatoblastoma and hepatocarcinoma in infancy and childhood. Cancer 1967;20:396-422.

25.- Leuschner I, Harris D, Schmidt D. The association of hepatocellular carcinoma in childhood with Hepatitis B infection. Cancer 1988;62: 2363-2369. 